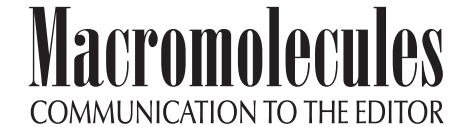

\section{Dynamics of Uniaxially Oriented Elastomers Using Broadband Dielectric Spectroscopy}

\author{
HyungKi Lee, ${ }^{\dagger}$ Daniel Fragiadakis, ${ }^{\ddagger}$ Darren Martin,,${ }^{\S}$ \\ Anthony Milne, ${ }^{\perp}$ John Milne,${ }^{\perp}$ and James Runt ${ }^{*}$,
}

${ }^{\dagger}$ Department of Materials Science and Engineering, Penn State University, University Park, Pennsylvania 16802, *Naval Research Lab, Washington, D.C. 20375, ${ }^{\S}$ Australian Institute of Bioengineering \& Nanotechnology, University of Queensland, St. Lucia, QLD 4072, Australia, and ${ }^{\perp}$ Milne Corp, Brisbane, QLD, Australia

Received February 10, 2010

Revised Manuscript Received March 9, 2010

The molecular mobility of chemically cross-linked and thermoplastic elastomers is of utmost importance in establishing physical properties for uses ranging from automotive tires and shoe soles to more sophisticated aerospace and biomedical applications. In many of these applications, network chain dynamics under external stresses/strains are critical for determining ultimate performance. It is well established that $T_{\mathrm{g}}$ (or $T_{\alpha}$, the dynamic $T_{\mathrm{g}}$ ) and the breadth of the segmental $(\alpha)$ relaxation time distribution increase with increasing cross-link density for chemically cross-linked polymers. For elastomers, there are considered to be two contributions important in determining the change in $T_{\alpha}$ upon uniaxial extension: a reduction in conformational entropy, encouraging an increase in $T_{\alpha}$, and a volume increase on uniaxial deformation, leading to a reduction in $T_{\alpha}$ (nonideal networks are compressible to some degree). ${ }^{1,2}$

Considering the importance of mechanical deformation in elastomer applications, there have been relatively few previous investigations of mechanical strain on chain dynamics, e.g., ${ }^{3,4}$ even fewer using broadband dielectric spectroscopy, and none on thermoplastic elastomers. ${ }^{5-8}$ This is despite the fact that modern broadband dielectric spectroscopy is a powerful tool for the investigation of material dynamics over very wide frequency and temperature ranges.

In this Communication, we report the findings of our initial dielectric spectroscopy investigation of the role of uniaxial extension on the relaxation behavior of cross-linked polyisoprene and segmented polyurethane elastomers. A Novocontrol GmbH Concept 40 broadband dielectric spectrometer was used to measure dielectric permittivity and loss over a broad range of temperatures and frequencies.

Synthetic polyisoprene [PI, NIPOL-IR2200 (Zeon Chemicals), $98.5 \%$ cis-1,4 content] was cross-linked with dicumyl peroxide (Sigma-Aldrich) at $180^{\circ} \mathrm{C}$ for $4 \mathrm{~min}$ in an aerated oven. Crosslink density was determined by measuring the swelling ratio in toluene and the Flory-Rehner equation. ${ }^{9}$ A poly(tetramethylene oxide) (PTMO, MW 1000) soft segment polyurethane (PU) with $32.5 \mathrm{wt} \%$ of hard segments [4,4'-diphenylmethane diisocyanate (MDI) and 1,4-butanediol (BDO)] was supplied by AorTech Biomaterials (Scoresby, QLD, Australia) in film form.

For the uniaxial deformation experiments, the stretching rig shown in Figure 1 was developed in order to allow convenient loading of deformed specimens directly into the dielectric spectrometer. This rig has three essential parts: sample clamper, electrode,

\footnotetext{
*To whom correspondence should be addressed. E-mail: runt@
} matse.psu.edu. and stretching knob. A sample strip $(10 \mathrm{~mm} \times 80 \mathrm{~mm})$ was placed along the groove on the rig and drawn to a specific extension ratio $(\lambda)$. An electrode was positioned in the middle of the strained sample and clamped. The strained sample-electrode assembly was then separated from the rig and dried in vacuum for $24 \mathrm{~h}$ before measurement.

The influence of uniaxial extension on the dynamics of the cross-linked PI (cross-link density $=6.1 \times 10^{-5} \mathrm{~mol} / \mathrm{cm}^{3}$ ) is displayed in Figure 2. Two relaxation processes are observed in dielectric spectra: the segmental (see Figure 2a) and normal mode (not shown) relaxations. The latter is observed in cross-linked polymers with type A dipoles, despite the fact that the network chains are fixed by cross-linking, due to the contribution of dangling chain ends. ${ }^{10}$ The relaxation times of both processes, shown in Figure 2b, are unaffected by uniaxial deformation. These results are in agreement with previous experimental results on PI and similar cross-linked elastomers. ${ }^{5-8}$ Even at higher extension ratios $(\lambda>4)$, at which strain-induced crystallization was detected by $\mathrm{X}$-ray diffraction experiments, no meaningful change in the segmental dynamics was observed.

The segmented polyurethane with $32.5 \mathrm{wt} \%$ MDI-BDO hard segments undergoes microphase separation when cooled from the one phase melt, resulting in hard domains phase separated from a low- $T_{\mathrm{g}}$ phase, the latter consisting of PTMO soft segments and some, presumably short, dissolved hard segments. ${ }^{11,12}$ Two relaxations are observed in the temperature range of the current experiments. The low-temperature $\beta$ process follows an Arrhenius form (see Figure $3 \mathrm{~b}$ ) with an activation energy of $\sim 35 \mathrm{~kJ} / \mathrm{mol}$ and is associated with local motion of the soft segments. ${ }^{13}$ The higher temperature $\alpha$ relaxation follows a VFT temperature dependence, extrapolates to the soft phase $T_{\mathrm{g}}$ at $\tau=100 \mathrm{~s}$, and is associated with the soft phase segmental relaxation. The local $\beta$ process is unaffected by uniaxial deformation up to $\lambda=4$. However, unlike cross-linked PI, the $\alpha$ process of the segmented polyurethane varies substantially: it moves to lower frequencies (Figure 3a) with increasing $\lambda$, accompanied by broadening of the relaxation (the Havriliak - Negami broadening shape parameter for the fitted relaxation decreases from 0.42 to 0.24$)$ and a decrease in the relaxation strength $[=4.1(\lambda=1)$ to $2.7(\lambda=4)]$. Extrapolation of the segmental relaxation times yields estimates of the soft phase $T_{\mathrm{g}}$ for the various deformed states (Table 1), and this increases by about $25^{\circ} \mathrm{C}$ over that of the undeformed PU segmented elastomer for $\lambda=4$. The sensitivity of the segmental dynamics for the PU relative to the PI under consideration here is due to the difference in effective cross-link density. Since many (although not all) hard segments reside in hard domains, the effective cross-link density for the segmented PU is on the order of 1000 (the molecular weight of the soft segments) while that for the cross-linked PI is 33000 and results in significant constrained mobility of PU soft phase segments under deformation.

Structural characterization of the PU microphase-separated morphology as a function of $\lambda$ is currently underway to provide a complete basis for understanding the changes in dynamics, although some general features are expected based on previous research on similar PUs. ${ }^{14-16}$ Segments in the soft phase (soft segments and dissolved hard segments) will first become oriented in the deformation direction due to the lower modulus of the soft phase and stress will be ultimately be exerted on the hard domains. If anisotropic in shape, the hard domains are expected to align with their long axis in the deformation direction. 


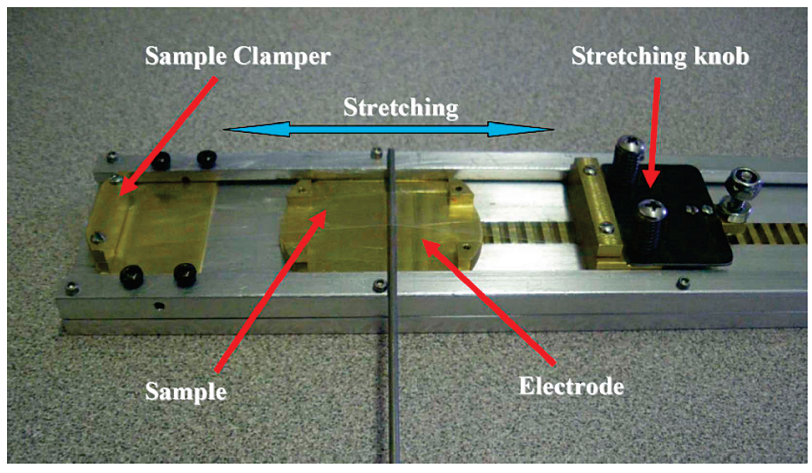

Figure 1. Uniaxial deformation rig.
Hard domains may then fragment and/or plastically deform with increasing extension, and if the soft segments are sufficiently regular and of high enough molecular weight, strain-induced crystallization may also occur (this was not for the PTMO copolymer investigated here up to $\lambda=4$ ).

Table 1. VFT Fitting Parameters and Dynamic Fragility Index $(m)$ of the Segmented Polyurethane at Various Extension Ratios

\begin{tabular}{lllll}
\hline & unstretched & \multicolumn{1}{c}{$\lambda=2$} & \multicolumn{1}{c}{$\lambda=3$} & \multicolumn{1}{c}{$\lambda=4$} \\
\hline$\tau_{0}(\mathrm{~s})$ & $1.85 \times 10^{-6}$ & $2.23 \times 10^{-6}$ & $1.3 \times 10^{-6}$ & $4.18 \times 10^{-6}$ \\
$E_{\mathrm{a}}(\mathrm{eV})$ & 0.067 & 0.064 & 0.064 & 0.038 \\
$T_{0}(\mathrm{~K})$ & 171 & 175 & 181 & 215 \\
$T(\tau=100 \mathrm{~s})$ & 215 & 217 & 222 & 241 \\
fragility $m$ & 57 & 61 & 70 & 93
\end{tabular}

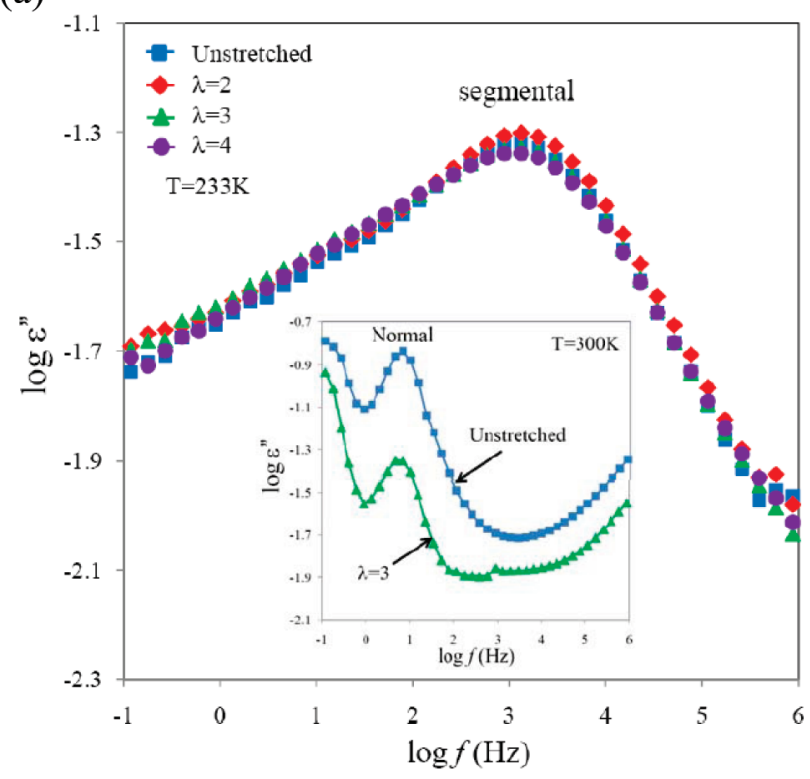

(b)

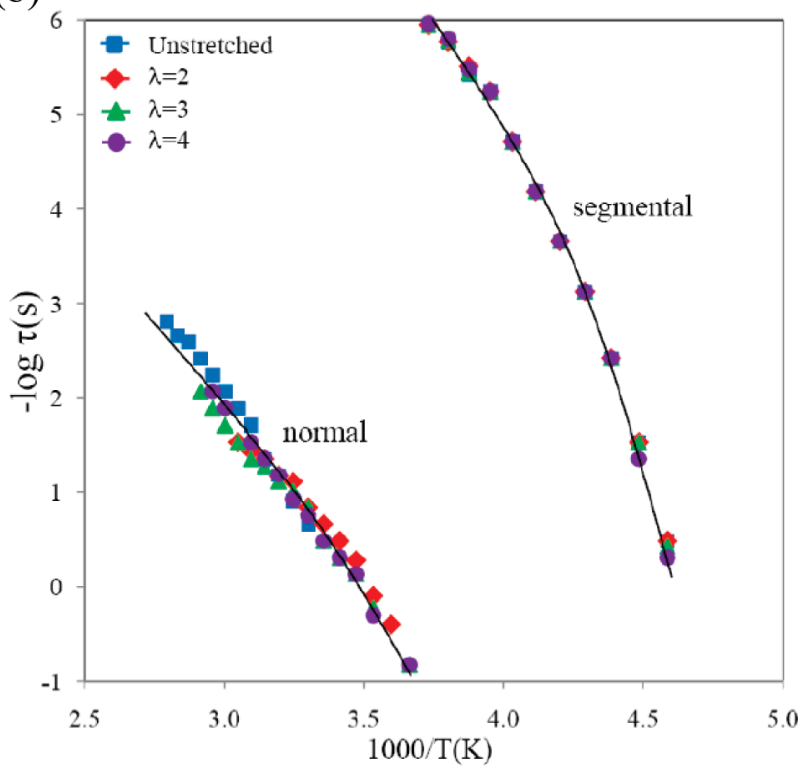

Figure 2. (a) Dielectric loss factor for the segmental processes and (b) VFT plot of relaxation processes as a function of extension ratio for the crosslinked polyisoprene (cross-linking density $=6.1 \times 10^{-5} \mathrm{~mol} / \mathrm{cm}^{3}$ ). Inset in (a) depicts the effect of deformation on the normal mode process in the undeformed state and for $\lambda=3$, at $T=300 \mathrm{~K}$.

(a)

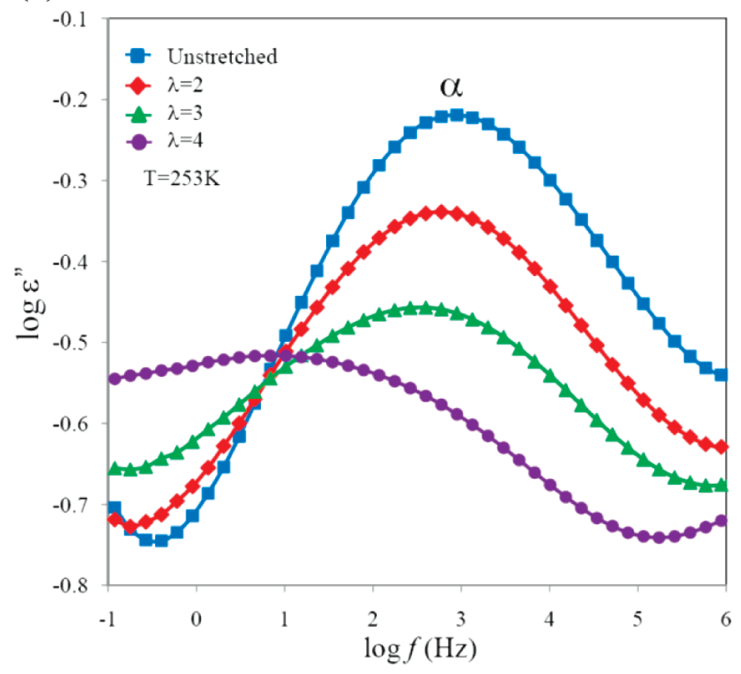

(b)

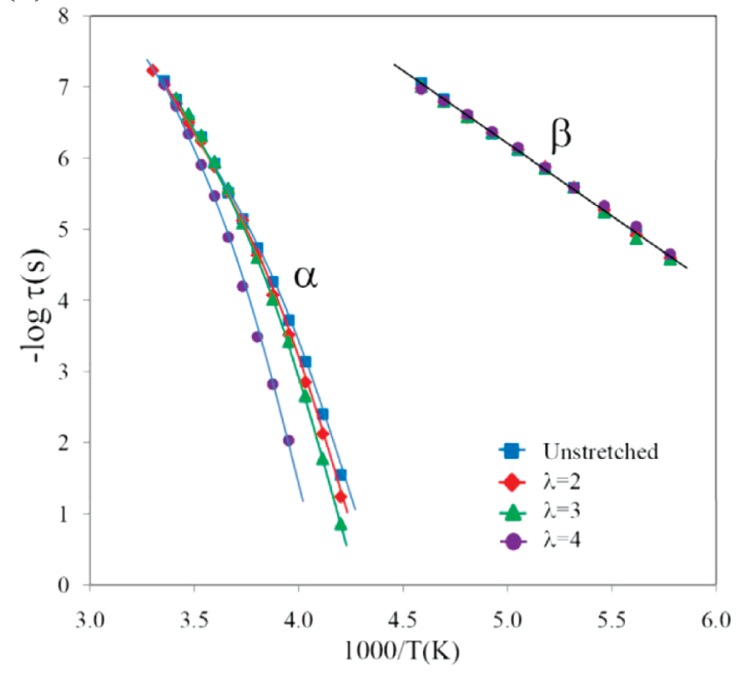

Figure 3. (a) Dielectric loss factor at $253 \mathrm{~K}$ for the $\alpha$ processes in the frequency domain and (b) VFT plot of relaxation processes as a function of extension ratio for a segmented polyurethane with $32.5 \mathrm{wt} \%$ hard segments. 
The dynamic fragility $(m)$ of soft phase glass formation can be determined as a function of extension ratio using ${ }^{17}$

$$
m=\left.\frac{\mathrm{d} \log \tau}{\mathrm{d}\left(T_{\alpha} / T\right)}\right|_{T=T_{\mathrm{g}}}
$$

where $\tau$ is the structural relaxation time. A stronger deviation from Arrhenius behavior corresponds to a more fragile system. As shown in Table 1, the dynamic fragility increases gradually from 57 in the undeformed state to 70 at $\lambda=3$ and then jumps to 93 when $\lambda$ reaches 4 . Fragility is generally correlated with the degree of intermolecular coupling, ${ }^{18}$ and as intermolecular coupling becomes stronger the material becomes more fragile. Fragility has been demonstrated to increase with increasing chemical cross-linking ${ }^{19,20}$ as well as intermolecular hydrogen bonding. ${ }^{21}$ The general increase in $m$ with $\lambda$ is in keeping with the increase in $T_{\mathrm{g}}{ }^{22}$ arising from increasing constraints on soft phase segments, and a more comprehensive discussion will be provided in a following paper.

In summary, the segmental process of lightly cross-linked PI is not affected by uniaxial deformation up to $\lambda=4$, while that of a representative microphase-separated PU thermoplastic elastomer varies significantly in location, strength, and relaxation breadth with increasing $\lambda$. Although the details of the variation of the microphase-separated morphology are still under investigation, the behavior is in keeping with the larger effective crosslink density of the segmented PU and the resulting constrained mobility of soft phase segments under stress.

Acknowledgment. The authors gratefully acknowledge the support of the National Science Foundation, Polymers Program, under Grant DMR-0907139.

\section{References and Notes}

(1) Eisenberg, A.; Shen, M. Rubber Chem. Technol. 1968, 43, 156.

(2) Sharf, M. A.; Mark, J. E. Rubber Chem. Technol. 1980, 53, 982.

(3) Sanz, E.; Riande, E.; Mark, J. E. Macromolecules 1984, 17, 899.

(4) Munch, E.; Pelletier, J. M.; Sixou, B.; Vigler, G. Polymer 2006, 47, 3477.

(5) Ozkazanc, E.; Guney, H. Y.; Oskay, T.; Tarcan, E. J. Appl. Polym. Sci. 2008, 109, 3878.

(6) Diaz-Calleja, R.; Riande, E.; Guzman, J. J. Polym. Sci., Polym. Phys. Ed. 1990, 28, 1551.

(7) Nakajima, K.; Naoki, M.; Nose, T. Polym. J. 1978, 10, 307.

(8) Naoki, M.; Nakajima, K.; Nose, T.; Hata, T. Polym. J. 1974, 4, 283.

(9) Flory, P. J.; Rehner, J. J. Chem. Phys. 1943, 11, 521.

(10) Poh, B. T.; Adachi, K.; Kotaka, T. Macromolecules 1987, $20,2574$.

(11) Hernandez, R.; Weksler, J.; Padsalgikar, A.; Choi, T.; Angelo, E.; Lin, J. S.; Xu, L.-C.; Siedlecki, C. A.; Runt, J. Macromolecules 2008, 41, 9767.

(12) Koberstein, J. T.; Stein, R. S. J. Polym. Sci., Polym. Phys. 1983, 21, 1439.

(13) McCrum, N. G.; Read, B. E.; Williams, G. Anelastic and Dielectric Effects in Polymeric Solids; Dover Publications: New York, 1991.

(14) Unsal, E.; Yalcin, B.; Yilgor, I.; Yilgor, E.; Cakmak, M. Polymer 2009, 50, 4644

(15) Laity, P. R.; Taylor, J. E.; Wong, S. S.; Khunkamchoo, P.; Norris, K.; Cable, M.; Andrews, G. T.; Johnson, A. F.; Cameron, R. E. Polymer 2004, 45, 5215-5232.

(16) Finnigan, B.; Jack, K.; Campbell, K.; Halley, P.; Truss, R.; Casey, P.; Cookson, D.; King, S.; Martin, D. Macromolecules 2005, 38, 7386.

(17) Bohmer, R.; Angell, C. A. Phys. Rev. B: Condens. Matter 1992, 45, 10091.

(18) Angell, C. A. Science 1995, 267, 1924.

(19) Kramarenko, V. Y.; Ezquerra, T. A.; Sics, I.; Balta' Calleja, F. J.; Privalko, V. P. J. Chem. Phys. 2000, 113, 447.

(20) Roland, C. M. Macromolecules 1994, 27, 4242.

(21) Zhang, S. H.; Painter, P. C.; Runt, J. Macromolecules 2002, 35, 9403.

(22) Qin, Q.; McKenna, G. B. J. Non-Cryst. Solids 2006, 352, 2977. 